\title{
Proteasome Inhibitors Affect Appressorium Formation and Pathogenicity of the Rice Blast Fungus, Magnaporthe oryzae
}

\author{
Yiming Wang ${ }^{1, \dagger}$, Sang Gon Kim ${ }^{2, \dagger}$, Jingni Wu $\mathbf{u}^{1}$, Seok $\mathbf{Y u}^{2}$, Kyu Young Kang ${ }^{1,2 *}$ and Sun Tae Kim ${ }^{3 *}$ \\ ${ }^{1}$ Division of Applied Life Science (BK21 program), Gyeongsang National University, Jinju 660-701, Korea \\ ${ }^{2}$ Plant Molecular Biology and Biotechnology Research Center, Gyeongsang National University, Jinju 660-701, Korea \\ ${ }^{3}$ Department of Plant Bioscience, Pusan National University, Miryang 627-706, Korea \\ (Received on May 6, 2011; Revised on June 4, 2011; Accepted on June 14, 2011)
}

Previously, we identified the $20 S$ proteasome $\alpha$-subunit of Magnaporthe oryzae (M. oryzae) induced during appressorium formation, and detected an increase in multiple protein ubiquitination during the early appressorium formation process (Kim et al., 2004). In this study, we further attempted to determine whether the proteasome is involved in the appressorium formation of $M$. oryzae both in vitro and in planta, using proteasome inhibitors. A significant increase in $20 \mathrm{~S}$ proteasome during fungal germination and appressorium formation was observed using Western blot analysis with $20 \mathrm{~S}$ proteasome antibody, demonstrating that proteasomemediated protein degradation was involved in appressorium formation. Pharmacological analysis using proteasome inhibitors, MG-132, proteasome inhibitor I (PI) and proteasome inhibitor II (PII) revealed that germination and appressorium formation were delayed for 4 to $6 \mathrm{~h}$ on rice leaf wax-coated plates. Similarly, the treatment of proteasome inhibitors with fungal conidia on the rice leaf surface delayed appressorium formation and host infection processes as well. Additionally, fungal pathogenicity was strongly reduced at 4 days' postfungal infection. These data indicated that the fungal $20 \mathrm{~S}$ proteasome might be involved in the pathogenicity of $M$. oryzae by the suppression of germination and appressorium formation.

Keywords : Appressorium formation, Magnaporthe oryzae, Pathogenicity, Proteasome inhibitor, 20S proteasome

Rice blast fungus, which is caused by the filamentous fungus Magnaporthe oryzea, is the most serious disease in

\footnotetext{
*Corresponding authors.

ST Kim

Phone) +82-55-350-5505, FAX) +82-55-350-5509

E-mail) stkim71@pusan.ac.kr

KY Kang

Phone) +82-55-772-1961, FAX) +82-55-772-1969

E-mail: kykang@gnu.ac.kr

†These authors contributed equally to this work
}

food-source cultivar rice. M. oryzae conidia begin their germination after landing on the hard cell surfaces of the host, given appropriate temperature and humidity conditions. Then, an infection-related structure, the appressorium, differentiates from the germ tube tip within a few hours (Hoch et al., 1987). This dome-shaped cell could accumulate proteins and enzymes, and generate mechanical force to break down the cell wall structure of the host (Howard et al., 1991; Park et al., 2009). This fungal appressorium formation process is the initial stage in host penetration, which is essential for host infection. Previous reports have demonstrated that chemicals or physical signals could interfere with fungal germination and appressorium formation, including cutin monomers, nitrogen or carbon starvation stress, surface hardness, hydrophobicity of the leaf cuticle, and exogenous cAMP (Dickinson et al., 1977; Dick et al., 1979; Gilbert et al., 1996; Jelitto et al., 1994; Lee et al., 1993; Lee et al., 1994; Xiao et al., 1994). cAMP, a signal compound, induces appressorium formation on a non-inductive plate (Lee et al., 1993). To evaluate the molecular mechanisms of fungal appressorium formation, serial analysis of gene expression (SAGE) was carried out to evaluate the formation of $M$. oryzae appressoria triggered by cAMP (Irie et al., 2003). Twenty eight tags were highly induced during the appressorium formation process, including pathogenicity-related genes, subtilisin-like protease, MPG1, and MAS. Later, a proteomics analysis was conducted to identify the differentially-regulated proteins involved in M. oryzae appressorium formation (Kim et al., 2004). Changes in protein patterns during rapid progress in germination and appressorium formation were observed under inductive and non-inductive conditions. The major proteins significantly induced in appressorium development were the $20 \mathrm{~S}$ proteasome family proteins (Kim et al., 2004). The 20S proteasome, a subunit of the $26 \mathrm{~S}$ proteasome, was initially detected in Thermoplasma acidophilum (Dahlmann et al., 1989). The proteasomes are large multi subunit protease complexes, which operate in particular via the ubiquitin-proteasome pathway, and selectively degrade intra- 
cellular proteins in eukaryotic cells (Baumeister et al., 1998). The proteolytic core of this large $(2,000 \mathrm{kDa})$ complex is formed by the $20 \mathrm{~S}$ proteasome, a barrel-shaped structure shown to be composed of four rings, each containing seven subunits $\left(\alpha_{7} \beta_{7} \beta_{7} \alpha_{7}\right)$. The $\alpha$-subunits make up the two outer rings and the $\beta$-subunits comprise the two inner rings of the stack (Lowe et al., 1995). The 26S proteasome is known to perform a critical function in cell cycle progresses in various eukaryotic cells and plants by mediating protein degradations (Kawahara et al., 1992; King et al., 1996; Yanagawa et al., 2002; Kurepa et al., 2009; Skaar et al., 2009). They also involve multiple specific functions including cell signaling, gene regulation, selective elimination of abnormal proteins, programmed cell death, and substrate flux through metabolic pathways (George et al., 1999; Rock et al., 1999). Proteasome catalytic activity may also be influenced by a variety of environmental factors and pathological states (Shah et al., 2001). However, the biological and cellular functions of proteasome in fungal phytopathogens including $M$. oryzae remain poorly understood. During the last several years, importance of massive protein degradation has showed closely relation with fungal infection, especially appressorium formation (Kershaw et al., 2009). Similar results were also described in the infection structure-specific development of other pathogens (Nguyen et al., 2011; Oku et al., 2010; Veneault-Fourrey et al., 2006). However, no information is available of the proteasome mediated protein degradation during fungal and host interaction. In this study, a pharmacological assay was conducted using various proteasome inhibitors to determine whether the $20 \mathrm{~S}$ proteasome may be involved in appressorium formation and fungal pathogenicity. The delay of fungal germination and appressorium formation was noted in the presence of the proteasome inhibitors MG-132 and PII under both in vitro and in planta conditions. The pathogenicity of $M$. oryzae was also reduced by those inhibitors. In this study, we suggest for the first time that the 20S proteasome may be involved in appressorium formation and fungal pathogenicity.

\section{Materials and Methods}

Proteasome inhibitors. The proteasome inhibitors, MG132 (benzyloxycarbonyl-leucyl-leucyl-leucinal: Z-LLL$\mathrm{CHO}$ ), proteasome Inhibitor I (N-carbobenzoxy-L- $\gamma$-t-butyl lglutamyl-L-alanyl-L-leucinal: Z-lle-Glu(OtBu)-Ala-Leu$\mathrm{CHO}$ ), and proteasome inhibitor II (benzyloxycarbonylleucyl-leucyl-phenylalaninal: Z-LLF-CHO) were purchased from Calbiochem (San Diego, CA, USA). These proteasome inhibitors were dissolved in DMSO and small aliquots were stored at $-20{ }^{\circ} \mathrm{C}$ until treatment. Proteasome inhibitors dissolved in DMSO were applied to the spores at final concentrations of $20 \mu \mathrm{M}$ or $40 \mu \mathrm{M}$. The final concentration of DMSO was $0.5-1 \%$ in the $M$. oryzae conidia.

Fungal strains, growth conditions and plant inoculation. The conidia of the rice blast fungus (race KJ301), which is compatible with rice cv. Jinheung, was used in this study. The conidia were prepared in accordance with the method described by Kim et al. (2004). In brief, the conidia were incubated on rice bran agar medium ( $25 \mathrm{~g}$ of rice bran, $1 \mathrm{~g}$ of sucrose and $20 \mathrm{~g}$ of agar in 1 liter deionized water, autoclaved) in darkness for 3 days at $28^{\circ} \mathrm{C}$, and the aerial mycelia were removed with a sterilized loop and cultured under fluorescent light conditions. Six- or sevenday old conidia were harvested by distilled water containing $0.02 \%$ Tween-20, then filtered through Wypall L25 kimtowels (Kimberly-Clark, USA) to remove aerial mycelia and cell debris. The conidia were washed twice in distilled water containing $0.02 \%$ Tween-20. The conidia were determined using a hemocytometer, and then diluted to $5 \times 10^{5} / \mathrm{ml}$ with distilled water containing $0.02 \%$ Tween20. The prepared fungal conidia were then spread using a hand sprayer on wax-coated plates and leaves. For inoculation on detached leaves, the conidia were dropped onto the leaf surface.

Rice preparation and treatment. The dehulled seeds of rice (Oryza sativa cv. Dongjin) were washed for $5 \mathrm{~min}$ with $70 \%$ ethanol, followed by a second washing for $30 \mathrm{~min}$ in $3 \%$ sodium hydrochloride. The seeds were rinsed at least five times with distilled water, followed by $48 \mathrm{~h}$ of imbibition in distilled water at $4{ }^{\circ} \mathrm{C}$. The imbibed seeds were transferred onto $0.8 \%$ phytagel plates and incubated in a light chamber at $28^{\circ} \mathrm{C}$. The germinated rice seedlings were transferred into soil mixture, and cultured in a greenhouse. Four- to six-leaf stage rice leaves were used for chemical or fungal treatments. The rice leaves were cut and attached on Grade No. 2 filter paper (Waterman, USA), and placed on moistened glass plates. Droplets of $15 \mu \mathrm{l}$ of spore suspensions $\left(1 \times 10^{5} / \mathrm{ml}\right)$ with $40 \mu \mathrm{M}$ of inhibitors were applied to inductive plates and the surfaces of detached rice leaves, then incubated for 6 and $8 \mathrm{~h}$ at $25^{\circ} \mathrm{C}$.

Preparation of rice wax-coated plates. The surface wax of rice leaves was extracted by submersion in organic solvent (petroleum ether:chloroform:benzene $=3: 1: 0.1$ ) for $30 \mathrm{~min}$. The wax solution was evaporated under a rotary evaporator. Plates in hydrophobic inductive condition were coated with the rice leaf extracts at a $1.2 \mu \mathrm{g} / \mathrm{cm}^{2}$ wax concentration. The wax coated plates were rinsed with methanol and sterilized water, then air-dried. Plates in hydrophilic non-inductive condition were cleaned up for 1 
h using cleaning solution ( $0.1 \mathrm{M}$ potassium dichromate/0.1 $\mathrm{N} \mathrm{H}_{2} \mathrm{SO}_{4}$ solution), followed by several changes and washings with sterilized distilled water.

Western blot analysis. Total protein $(20 \mu \mathrm{g})$ was extracted with $\mathrm{Mg} / \mathrm{NP}-40$ extraction buffer as previously described (Kim et al., 2004). The total proteins were separated by $12 \%$ SDS-PAGE, and then transferred to a PVDF membrane (Millipore, Massachusetts, USA) using a semidry electrophoretic apparatus (Hoefer, Holliston, MA). The blotted membrane was blocked for $4 \mathrm{~h}$ at room temperature in $1 \times$ TTBS buffer $(50 \mathrm{mM}$ Tris-HCl, pH 8.2, 0.1\% v/v Tween 20 , and $150 \mathrm{mM} \mathrm{NaCl}$ ) with $7 \%(\mathrm{w} / \mathrm{v})$ skimmed milk (BD Bio, USA), and incubated for $2 \mathrm{~h}$ after the addition of primary antibody (diluted to $1: 10,000)$. The membranes were sequentially incubated with anti-ubiquitin or anti-20S proteasome $\alpha$-subunits from Methanosarcina thermophila (Calbiochem, CA, USA), then washed 3 times for $15 \mathrm{~min}$ each with $1 \times$ TTBS. A secondary anti-rabbit IgG antibody conjugated with horseradish peroxidase diluted $1: 10,000$ in $1 \times$ TTBS was used for immunodetection. Signals were detected via ECL (Perkin Elmer Life Sciences, Boston, MA).

Southern blot analysis. Same amount of genomic DNA $(10 \mu \mathrm{g})$ from rice blast fungus was digested with EcoRI, EcoRV, KpnI and XhoI, respectively. Digested DNA was separated by electrophoresis on a $0.8 \%$ (w/v) agarose gel, and blotted onto a nylon membrane (Zeta Probe, BIORAD, USA). The blotted membrane was hybridized with $\left[\alpha-{ }^{32} \mathrm{P}\right] \mathrm{dCTP}$ labeled $20 \mathrm{~S}$ proteasome full length probe. The hybridized membrane was incubated at $65^{\circ} \mathrm{C}$ overnight and washed with $2 \times$ SSC with $0.1 \%$ (w/v) SDS at room temperature for $15 \mathrm{~min}$, and three times in $0.2 \times$ $\mathrm{SSC}$ with $0.1 \%(\mathrm{w} / \mathrm{v}) \mathrm{SDS}$ at $65^{\circ} \mathrm{C}$ for $15 \mathrm{~min}$. The membrane was exposed to an X-ray film after $24 \mathrm{~h}$ keeping at $-80{ }^{\circ} \mathrm{C}$.

Detection of fungal germination and appressorium formation on rice leaves. The infected leaves were fixed with ethanol:acetic acid (2:1), and stained with $1 \% \mathrm{v} / \mathrm{v}$ acid fuchsin-lactophenol solution $(20 \%(\mathrm{v} / \mathrm{v})$ phenol, $20 \%(\mathrm{v} / \mathrm{v})$ lactic acid, $40 \%$ (v/v) glycerine) for $1 \mathrm{~min}$. To observe infected mycelium and callose formation via fluorescence microscopy, the leaves were preserved in $1 \mathrm{~N} \mathrm{KOH}$. The $\mathrm{KOH}$-preserved specimens were briefly autoclaved, rinsed with $0.067 \mathrm{M} \mathrm{K}_{2} \mathrm{HPO}_{4}$ at $\mathrm{pH} 9.0$, and stained with $0.05 \%$ aniline blue dye in $0.067 \mathrm{M} \mathrm{K}_{2} \mathrm{HPO}_{4}$ at $\mathrm{pH}$ 9.0. Three biological replicate assays were conducted for proteasome inhibitors and infection foci were analyzed after 3 days of incubation in a humidity chamber.

\section{Results and Discussion}

Expression of proteasome in $M$. oryzae during fungal germination and appressorium formation. Proteasome is a conserved protein in all plants, microbes, and animals. Here, we first compared the homology of the deduced amino acid sequences of the $20 \mathrm{~S}$ proteasome from M. oryzae with sequences from other species. Highly conserved domains were detected in all sequences (Fig. 1). The M. oryzae $20 \mathrm{~S}$ proteasome evidenced $58.6 \%, 58 \%, 54.7 \%$, and $91.3 \%$ homology with mouse, human, Arabidopsis, and Neurospora, respectively. It was also found to be highly homologous with Neurospora, indicating that the M. oryzae $20 \mathrm{~S}$ proteasome is tightly conserved with the fungi kingdom. The copy number of the $20 \mathrm{~S}$ proteasome in M. oryzae was confirmed via Southern blot analysis, resulting in at least two copies of $20 \mathrm{~S}$ proteasome in the fungal chromosome (Supplementary Fig. 1).

In an effort to determine whether the $20 \mathrm{~S}$ proteasome is involved in the fungal germination and appressorium

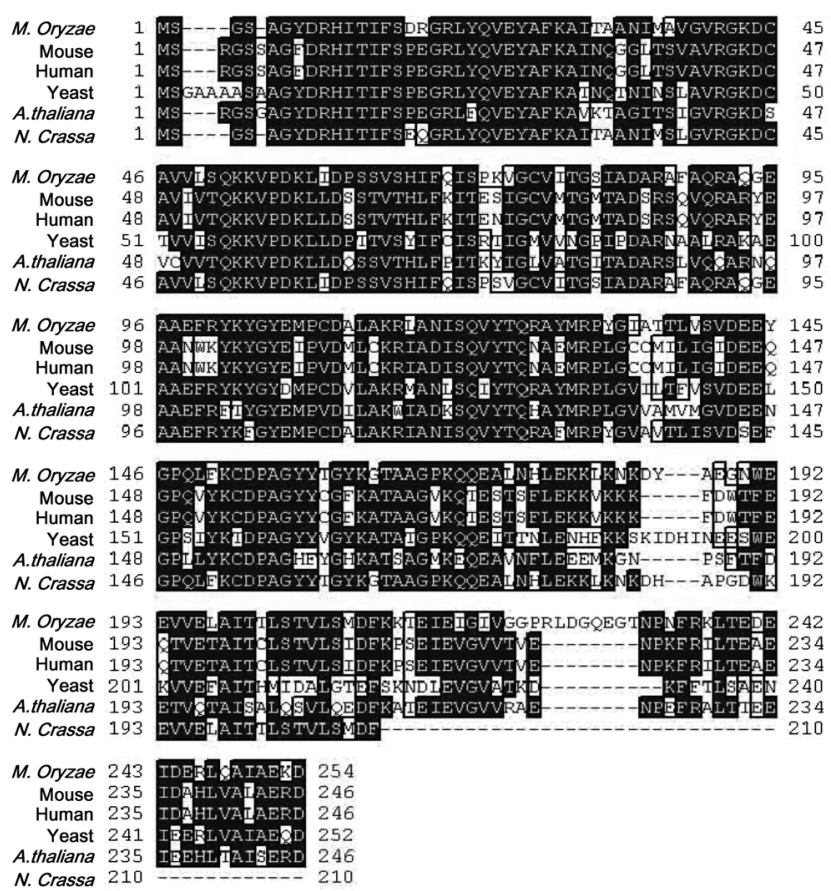

Fig. 1. Sequence conservancy between $M$. oryzae and other species. Amino acid sequences of $20 \mathrm{~S}$ proteasome from different species were obtained from the National Center for Biotechnology Information (NCBI) on line database (http:/www.ncbi.nlm.nih.gov/) with the accession number of XM_368091 (Magnaporthe oryzae, M. oryzae), NP_002782 (Mouse), P21243 (Human), P21243 (Yeast), AAC32054 (Arabidopsis thaliana, A. thaliana), and EAA28671 (Neurospora Crassa, N. Crassa). Alignment between the amino acid sequences was carried out using the BioEdit sequencing aligment Editor Program (v7.0.5), and the homology analyses were carried out using Victor NTI 7.0 software. 


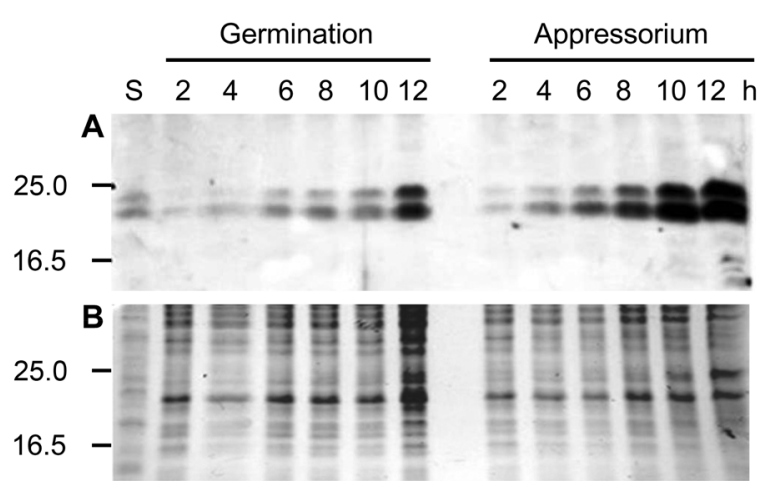

Fig. 2. Time-course accumulation of $20 \mathrm{~S}$ proteasome during fungal germination and appressorium formation processes. (A) Western blots were prepared from total proteins obtained from fungal conidia during germination and appressorium formation in samples cultured on hydrophilic surfaces, and blotted with the anti-20S proteasome $\alpha$-subunit. (B) SDS-PAGE was conducted using Coomassie Brilliant Blue.

formation processes, we analyzed the protein expression level of the 20S proteasome in a time-dependent manner. Western blot analysis was employed to detect the accumulation of $20 \mathrm{~S}$ proteasome using $20 \mathrm{~S}$ proteasome monoclonal antibody at $0,2,4,6,8,12 \mathrm{~h}$ after germination or appressorium formation. Germinated and appressoriumformed conidial protein extracts were separated via SDSPAGE and stained with Coommassie Brilliant Blue (CBB) (Fig. 2). A significant increase of $20 \mathrm{~S}$ proteasome was detected via Western blot analysis, revealing that the $20 \mathrm{~S}$ proteasome may be related closely with the fungal germination and appressorium formation processes (Fig. 2). Double bands with molecular weights of 21 and $24 \mathrm{kDa}$ may indicate different $20 \mathrm{~S}$ proteasome subunits. Similarly, results were detected in the previous proteomic analysis of M. oryzae during appressorium formation. Two subunits of the $20 \mathrm{~S}$ proteasome, designated $\alpha 4$ and $\alpha 6$, were increased during the appressorium formation process; the results showed that the $\alpha 4$ and $\alpha 6$ subunits evidenced different molecular weights on SDS-PAGE; this result was consistent with our previous results (Kim et al., 2004).

Protein ubiquitination and effect of proteasome inhibitors during appressorium formation. The protein degradation induced by the proteasome was mediated by the multiple ubiquitination of ubiquitin proteins (Clague et al., 2010). Therefore, we evaluated the protein ubiquitination occurring during appressorium formation using ubiquitin-specific antibody. An increase of multiple bands with different molecular weights was detected, suggesting a rapid protein ubiquitination was occurred during appressorium formation. Even more, some detected bands showed mono-/biubiquitination rather than poly-ubiquitination, implying that

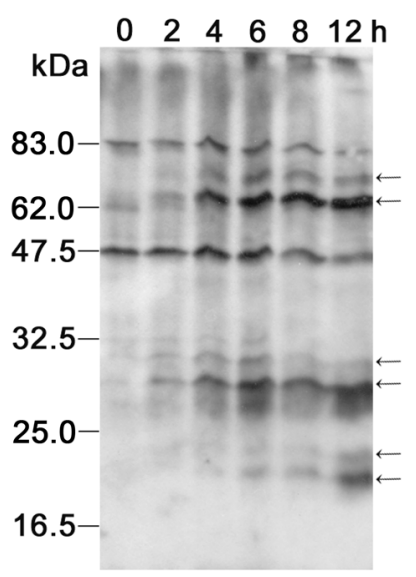

Fig. 3. Detection of protein ubiquitination during appressorium formation. The same amount of total proteins was extracted from M. oryzae at $0,2,4,6,8$ and $12 \mathrm{hr}$ after appressorium formation, and separated on $12.5 \%$ SDS-PAGE. Arrows indicated the ubiquitinated protein patterns.

the protein modification by ubiquitination may be as important as proteasome pathway mediated degradation during fungal appressorium formation (Fig. 3).

MG-132, proteasome inhibitor I (PI) and proteasome inhibitor II (PII) have been shown to be effective proteasome inhibitors in both fungi and mammals (Genin et al., 2010; Nandi et al., 2006). The proteasome inhibition activity during $M$. oryzae germination and appressorium formation was first confirmed. The M. oryzea spore suspensions treated with $40 \mu \mathrm{M}$ inhibitors were incubated on the hydrophobic surfaces of glass plates coated by rice leaf wax. The fungal morphologies were observed at $6 \mathrm{~h}$ and $8 \mathrm{~h}$ after incubation. Without inhibitor treatment, M. oryzae spores form the germ tube and the appressorium begins to differentiate at $6 \mathrm{~h}$, and mature appressoria were detected at $8 \mathrm{hr}$ (Fig. 4A). The application of the proteasome inhibitors MG-312, PI, and PII delayed the appressorium formation of M. oryzae on the wax-coated plates. Only the germ tube was detected at $6 \mathrm{~h}$ after treatment with those inhibitors, but no appressorium formation was observed to begin (Fig. 4A). At $8 \mathrm{~h}$, mature appressorium was detected in the PItreated fungus, but treatment with MG-132 and PII still suppressed appressorium formation (Fig. 4A). We further evaluated the inhibitory effects of MG-132 and PII at concentrations of $20 \mu \mathrm{M}$ and $40 \mu \mathrm{M}$ on conidial germination and appressorium formation (Fig. 4B, C). Without inhibitors, $60 \%$ of spores were germinated at $2 \mathrm{~h}$, whereas more than $90 \%$ were germinated at $4 \mathrm{hr}$ (Fig. 4B). After treatment with $20 \mu \mathrm{M}$ or $40 \mu \mathrm{M}$ MG-132, half the numbers of fungus were germinated at $4 \mathrm{~h}$, which represented a $2 \mathrm{~h}$ delay compared with the non-treated samples. After treatment with $20 \mu \mathrm{M}$ and $40 \mu \mathrm{M}$ proteasome inhibitor PII, the $50 \%$ germination rate was delayed by $5 \mathrm{~h}$ and $6 \mathrm{~h}$, respectively 


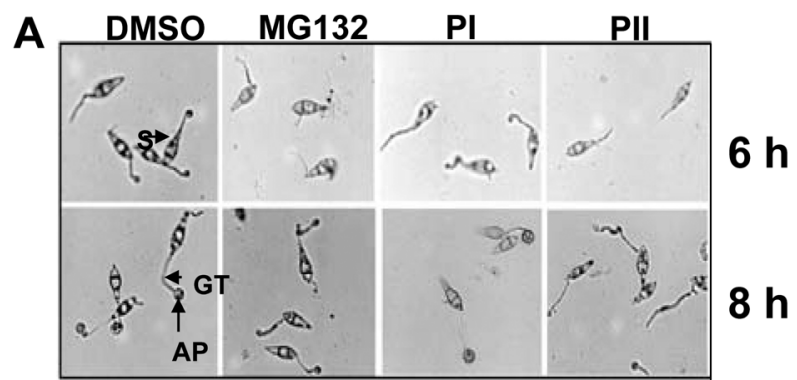

B

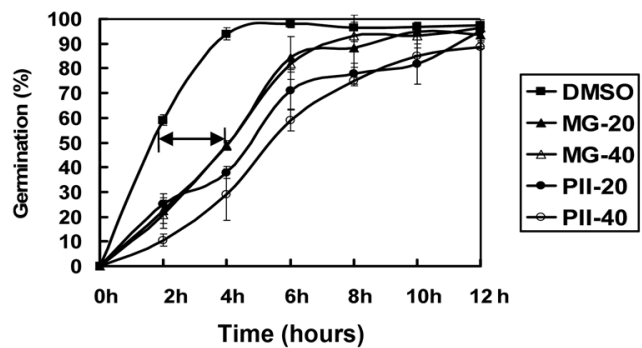

C

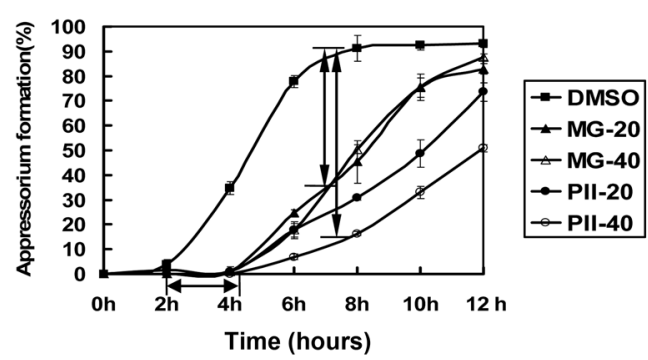

Fig. 4. The effect of proteasome inhibitors on M. oryzae spore germination and appressorium formation on rice leaf surface waxcoated glass plates. (A) Micrographs of conidia after $6 \mathrm{~h}$ and $8 \mathrm{~h}$ of incubation with $40 \mu \mathrm{M}$ proteasome inhibitors MG-132, PI, and PII. AP, appressorium; G, germ tube; S, spore. (B) Average rates of conidial germination and (C) appressorium formation in control (DMSO) and proteasome inhibitor treatment. At least 100 spores were counted per replicate. Data are the mean of three replications. MG-20, $20 \mu \mathrm{M}$ MG-132; MG-40, $40 \mu \mathrm{M}$ MG-132; PII-20, $20 \mu \mathrm{M}$ PII; PII-40, $40 \mu \mathrm{M}$ PII. Approximately 200 spores of each replicate were scored for germination or appressorium formation.

(Fig. 4B). The effects of MG-132 and PII on appressorium formation were then confirmed herein (Fig. 4C). No significant differences were detected with MG-132 treatment at concentrations of $20 \mu \mathrm{M}$ and $40 \mu \mathrm{M}$ until $12 \mathrm{hr}$. Half of the appressorium formation was detected at $8 \mathrm{~h}$ with MG-132 treatment. The proteasome inhibitor PII suppressed $70 \%$ of fungal appressorium formation at $8 \mathrm{~h}$ at a concentration of $20 \mu \mathrm{M}$, whereas $40 \mu \mathrm{M}$ of PII effected an $85 \%$ suppression of appressorium formation (Fig. 4C). These results showed that PII has the strongest inhibition activity, and the inhibition activity occurs in a dosage-dependent fashion. MG-132 at $20 \mu \mathrm{M}$ exhibits efficient inhibition activity against germination and appressorium formation. The pro- teasome inhibitor PII strongly suppressed appressorium formation. This inhibitor is unique, in its properties shared with the chymotrysin-like proteases, among the serine proteases in its ability to cleave proteins next to hydrophobic side chains (Marian et al., 1997). Among these treatments, serine protease inhibitors such as aprotinin, AEBSF, and PMSF exerted inhibitory effects on appressorium formation as well (data not shown). However, aspartic proteinase, pepstatin $\mathrm{A}$ and the cysteine protease inhibitor, leupeptin did not show evidence inhibitory effects against appressorium formation. This result indicates that specific proteolytic processes of serine protease may perform an important function in appressorium formation.

Pharmacological assay of appressorium formation on rice leaves. To evaluate the effects of those proteasome inhibitors on fungal infection in planta, we inoculated the M. oryzae spore on detached rice leaves supplemented with these proteasome inhibitors. Lactophenol staining demonstrated that $M$. oryzae conidia germinated on leaves treated with DMSO and all inhibitors at $6 \mathrm{~h}$ post-inoculation, but mature appressoria appeared only in the control leaves (Fig. 5A). Treatment with PI inhibitor suppressed appressorium formations for $6 \mathrm{~h}$, and MG132 and PII delayed appressorium formation for more than $8 \mathrm{~h}$. (Fig. 5A). The fungal infections were detected at $48 \mathrm{~h}$ post-inoculation with droplets of a $M$. oryzae conidial suspension of $1 \times 10^{5} / \mathrm{ml}$. The application of DMSO had no effect, and PI exerts a limited effect on fungal infection. However, the proteasome inhibitors, MG-132 and PII, strongly suppressed fungal

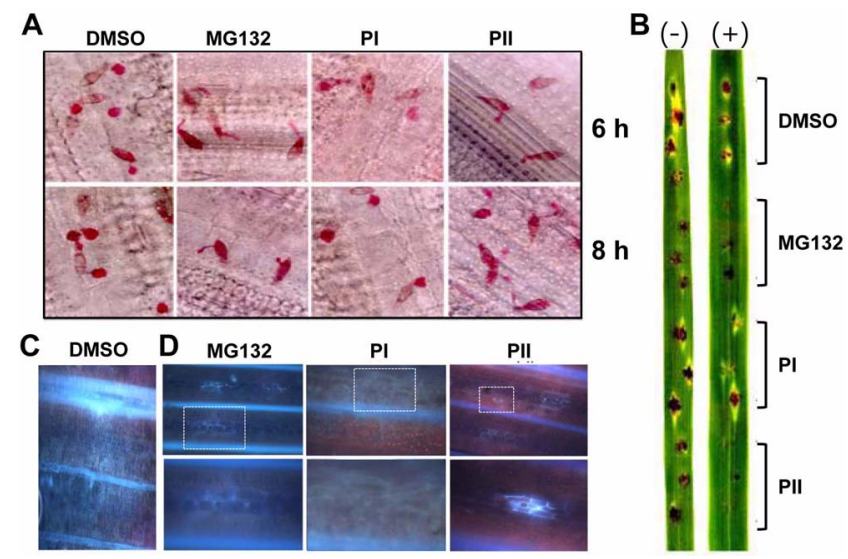

Fig. 5. In planta pathogenicity analysis of M. oryzae treated with proteasome inhibitors. (A) Fungal appressorium formation detected by lactophenol staining at $6 \mathrm{hr}$ and $8 \mathrm{hr}$ after inoculation. (B) Effects of proteasome inhibitors on the suppression of fungal infections at $48 \mathrm{~h}$ after inoculation. (-), negative control, leaf without chemical treatment; $(+)$, leaf treated with indicated chemicals. (C, D) Aniline blue fluorescent staining of appressorium and hyphae growing inside leaf cells at $48 \mathrm{~h}$ (top). A close-up view of fungal infection patterns (bottom). 
infection on the detached leaves (Fig. 5B). Less and none spread hyphae were detected after treatment with MG-132 and PII, respectively (Fig. 5B). It has been reported that callose deposition, which was detected by aniline blue and is induced by wounding stress or pathogen infection, was detected among the fungal infection patterns (Yim et al., 1998). The invading hyphae with serious branch spread were detected in infected leaves at $48 \mathrm{~h}$ with DMSO or PI treatment (Fig. 5C, D). The minimal spreading area and limited infection were demonstrated along with treatment of the MG-132 and PII inhibitors, respectively (Fig. 5D). Collectively, our results show that the proteasome inhibitors MG-132 and PII could reduce the fungal pathogenicity, demonstrating that the proteasome may be associated with fungal pathogenicity.

Proteasome inhibitor PII suppresses fungal pathogenicity in planta. The proteasome inhibitor PII exerted a profound suppressive effect against appressorium formation in $M$. oryzae on inductive glass plates and detached rice leaves. Therefore, we speculated that PII may suppress fungal pathogenicity in planta. Three biological replicate assays were conducted and infection foci were analyzed after 4 days of incubation in a humidity chamber. Without PII treatment, the leaves were heavily infected by M. oryzae (Fig. 6A). After spreading with $40 \mu \mathrm{M}$ PII and fungal spores, fewer infected foci appeared on the leaves after 4 days (Fig. 6A). PII suppressed $70 \%$ of fungal-infected foci relative to the non-treated tissues. An average of 35 infected foci were detected on control leaves, and less than 10 infected foci were detected on the PII-treated tissues (Fig. $4 \mathrm{~B})$. The expression level of the rice defense marker gene, PBZ1, was confirmed after treatment with proteasome inhibitor. No significant difference was observed by Northern blot analysis, suggesting that those inhibitors have limited effect on the host side (data not shown). These results imply that the biochemical PII may have some

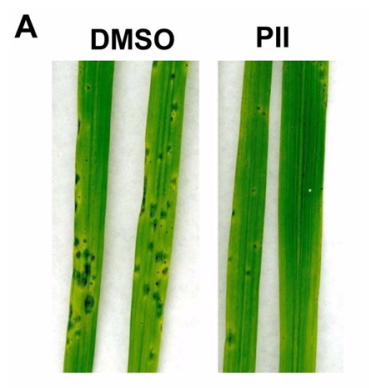

B

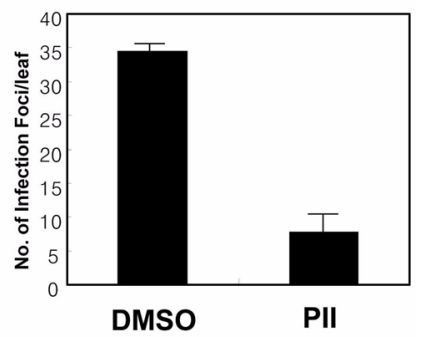

Fig. 6. Protection of rice leaves against $M$. oryzae by proteasome inhibitor II at 4 days after inoculation. (A) Rice leaf blast symptoms on control and PII-treated leaves were observed. (B) Average infection foci numbers on control and PII-treated leaves. All experiments were determined in at least three replications. ability to suppress $M$. oryzae pathogenicity via the inhibition of proteasome activity, but not by the activation plant immunity responses.

\section{Conclusions}

In this study, we determined that the treatment of proteasome inhibitors have the ability to suppress conidial germination, appressorium formation, and fungal pathogenicity under both in vitro and in planta conditions. This inhibition may be caused as the result of a defect in appressorium-mediated penetration, and may lead to the reduction or delay of fungal penetration. Ubiquitin genes are associated frequently with protein turnover via the ubiquitin proteasome pathway. $M$. oryzae is likely to be very metabolically active during the infection process, and thus these genes might play important roles; multiple genes are known to participate in appressorium formation and pathogenesis. The proteasome inhibitor PII exhibiting serine protease evidenced the strongest inhibition ability, and reduced the disease symptoms of $M$. oryzae on rice leaves (Fig. 5, 6). Overall, this study is, to the best of our knowledge, the first to demonstrate that the 20S proteasome may prove essential for appressorium formation and infectious growth in $M$. oryzae, and may help in the search for novel targets for fungal pathogenicity. However, the specific roles of the $20 \mathrm{~S}$ proteasome in the appressorium formation of $M$. oryzae remain to be clearly elucidated.

\section{Acknowledgments}

This work was supported by Basic Science Research Program through the National Research Foundation of Korea (NRF) funded by the Ministry of Education, Science and Technology (2011-0005370), by a grant from the NextGeneration BioGreen 21 Program (Plant Molecular Breeding Center, PJ008021), Rural Development Administration, Republic of Korea. Y. Wang and J. Wu were Supported by Scholarships from the BK21 Program.

\section{References}

Baumeister, W., Walz, J., Zuhl, F. and Seemuller, E. 1998. The proteasome: paradigm of self compartmentalizing protease. Cell 92:367-380.

Clague, M. J. and Urbé, S. 2010. Ubiquitin: same molecule, different degradation pathways. Cell 143:682-685.

Dahlmann, B., Kopp, F., Kuehn, L., Niedel, B., Pfeifer, G., Hegerl, R. and Baumeister, W. 1989. The multicatalytic proteinase (prosome) is ubiquitous from eukaryotes to archaebacteria. FEBS Lett. 251:125-131.

Dick, S. 1979. Growth of Erysiphe germinis on artificial membranes. Physiol. Plant Pathol. 15:219-221. 
Dickinson, S. 1977. Studies in the physiology of obligate parasitism X. induction of responses to a thigmotropic stimulus. Phytopathol. Z. 89:97-115.

Genin, E., Reboud-Ravaux, M. and Vidal, J. 2010. Proteasome inhibitors: recent advances and new perspectives in medicinal chemistry. Curr. Top. Med. Chem. 10:232-256.

George, N. D. and Clive, A. S. 1999. The proteasome, a novel protease regulated by multiple mechanisms. J. Biol. Chem. 27:22123-22126.

Gilbert, R. D., Johnson, A. M. and Dean, R. A. 1996. Chemical signals responsible for appressorium formation in the rice blast fungus. Physiol. Mol. Plant Pathol. 48:335-346.

Hoch, H. C. and Staples, R. C. 1987. Structural and chemical changes among the rust fungi during appressorium development. Annu. Rev. Phytopathol. 25:231-247.

Howard, R. J., Ferrari, M. A., Roach, D. H. and Money, N. P. 1991. Penetration of hard substrates by a fungus employing enormous turgor pressures. Proc. Natl. Acad. Sci. U. S. A. 88:11281-11284.

Irie, T., Matsumura, H., Terauchi, R. and Saitoh, H. 2003. Serial Analysis of Gene Expression (SAGE) of Magnaporthe grisea: genes involved in appressorium formation. Mol. Genet. Genomics 270:181-189.

Jelitto, T. C., Page, H. A. and Read, N. D. 1994. Role of external signals in regulating the pre-penetration phase of infection by the rice blast fungus, Magnaporthe grisea. Annu. Rev. Microbiol. 50:491-512.

Oku, M. and Sakai, Y. 2010. Peroxisomes as dynamic organelles: autophagic degradation. FEBS J. 277:3289-3294.

Park, J. Y., Jin, J., Lee, Y. W., Kang, S. and Lee, Y. H. 2009. Rice blast fungus (Magnaporthe oryzae) infects Arabidopsis via a mechanism distinct from that required for the infection of rice. Plant Physiol. 149:474-486.

Kawahara, H. and Yokosawa, H. 1992. Cell cycle-dependent change of proteasome distribution during embryonic development of the ascidian Halocynthia roretzi. Dev. Biol. 151:2733.

Kershaw, M. J. and Talbot, N. J. 2009. Genome-wide functional analysis reveals that infection-associated fungal autophagy is necessary for rice blast disease. Proc. Natl. Acad. Sci. U. S. A. 106:15967-15972.

Kim, S. T., Yu, S., Kim, S. G., Kim, H. J., Kang, S. Y., Hwang, D. H., Jang, Y. S. and Kang, K. Y. 2004. Proteome analysis of rice blast fungus (Magnaporthe grisea) proteome during appressorium formation. Proteomics 4:3579-3587.

King, R. W., Deshaies, R. J., Peters, J. M. and Kirschner, M. W. 1996. How proteolysis drives the cell cycle. Science 274: $1652-1659$.

Kurepa, J., Wang, S., Li, Y. and Smalle, J. 2009. Proteasome regu- lation, plant growth and stress tolerance. Plant Signal Behav. 4:924-927.

Lee, Y. H. and Dean, R. A. 1993. cAMP regulates infection structure formation in the plant pathogenic fungus Magnaporthe grisea. Plant Cell 5:693-700.

Lee, Y. H. and Dean, R. A. 1994. Hydrohobicity of contact surface induces appressorium formation in Magnaporthe grisea. FEMS Microbiol. Lett. 115:71-75.

Lowe, J., Stock, D., Jap, B., Zwickl, P., Baumeister, W. and Huber, R. 1995. Crystal structure of the 20S proteasome from the archaeon T. acidophilum at $3.4 \mathrm{~A}$ resolution. Science 268:533-539.

Marian, O., Christopher, C., Eleuteri, A. M., Kohanski, R., Kam, C. M. and Powers, J. C. 1997. Reactions of [14C]-3,4-Dichloroisocoumarin with subunits of pituitary and Spleen Multicatalytic Proteinase Complexes (proteasome). Biochemitry. 36: 13946-13953.

Nandi, D., Tahiliani, P., Kumar, A. and Chandu, D. 2006. The ubiquitin-proteasome system. J. Biosci. 31:137-55.

Nguyen, L. N., Bormann, J., Le, G. T., Stärkel, C., Olsson, S., Nosanchuk, J. D., Giese, H. and Schäfer, W. 2011. Autophagy-related lipase FgATG15 of Fusarium graminearum is important for lipid turnover and plant infection. Fungal Genet. Biol. 48:217-224.

Rock, L. and Goldberg, A. L. 1999. Degradation of cell proteins and the generation of MHC class 1-presented peptides. Аnпи. Rev. Immunol. 17:739-779.

Shah, S. A., Potter, M. W. and Callery, M. P. 2001. Ubiquitin proteasome pathway: implications and advances in cancer therapy. Surg. Oncol. 10:43-52.

Skaar, J. R. and Pagano, M. 2009. Control of cell growth by the SCF and APC/C ubiquitin ligases. Curr. Opin. Cell Biol. 21:816-824.

Veneault-Fourrey, C., Barooah, M., Egan, M., Wakley, G. and Talbot, N. J. 2006. Autophagic fungal cell death is necessary for infection by the rice blast fungus. Science 312:580-583.

Xiao, J. Z., Watanabe, T., Kamakura, T., Ohshima, A. and Yamaguchi, I. 1994. Studies on cellular differentiation of Magnoporthe grisea physicochemical aspects of substratum surface in relation to appressorium formation. Physiol. Mol. Plant Pathol. 44:227-236.

Yanagawa, Y., Hasezawa, S., Kumagai, F., Oka, M., Fujimuro, M., Naito, T., Makino, T., Yokosawa, H., Tanaka, K., Komamine, A., Hashimoto, J., Sato, T. and Nakagawa, H. 2002. Cell-cycle dependent dynamic change of $26 \mathrm{~S}$ proteasome distribution in tobacco BY-2 cells. Plant Cell Physiol. 43:604-613.

Yim, K. O. and Bradford, K. J. 1998. Callose deposition is responsible for apoplastic semipermeability of the endosperm envelope of muskmelon seeds. Plant Physiol. 118:83-90. 